Article

\title{
A Facile and Mild Synthesis of Trisubstituted Allylic Sulfones from Morita-Baylis-Hillman Carbonates
}

\author{
Lin Jiang ${ }^{1,2, *}$, Yong-Gen $\mathrm{Li}^{1}$, Jiang-Feng Zhou ${ }^{1}$, Yong-Ming Chuan ${ }^{1,2}$, Hong-Li $\mathrm{Li}^{1,2}$ and \\ Ming-Long Yuan ${ }^{1,2, *}$
}

1 Engineering Research Center of Biopolymer Functional Materials of Yunnan,

School of Chemistry and Biotechnology, Yunnan Minzu University, Kunming 650500, China;

E-Mails: lyg1990wsh@163.com (Y.-G.L.); zjf109lq@163.com (J.-F.Z.); chuan5211017@126.com (Y.-M.C.); honglili_1982@163.com (H.-L.L.)

2 Key Laboratory of Chemistry in Ethnic Medicinal Resources, State Ethnic Affairs Commission \& Ministry of Education, School of Chemistry and Biotechnology, Yunnan Minzu University, Kunming 650500, China

* Authors to whom correspondence should be addressed; E-Mails: jianglin_1981@163.com (L.J.); yml@vip.163.com (M.-L.Y.); Tel.: +86-871-6591-4825 (L.J. \& M.-L.Y.);

Fax: +86-871-6594-6825 (L.J. \& M.-L.Y.).

Academic Editor: Panayiotis A. Koutentis

Received: 1 April 2015 / Accepted: 4 May 2015 / Published: 7 May 2015

\begin{abstract}
An efficient and catalyst-free synthesis of trisubstituted allylic sulfones through an allylic sulfonylation reaction of Morita-Baylis-Hillman (MBH) carbonates with sodium sulfinates has been developed. Under the optimized reaction conditions, a series of trisubstituted allylic sulfones were rapidly prepared in good to excellent yields $(71 \%-99 \%)$ with good to high selectivity ( $Z / E$ from 79:21 to $>99: 1)$. Compared with known synthetic methods, the current protocol features mild reaction temperature, high efficiency and easily available reagents.
\end{abstract}

Keywords: Morita-Baylis-Hillman carbonate; allylic sulfone; trisubstituted alkene; allylic substitution 


\section{Introduction}

Morita-Baylis-Hillman (MBH) adducts and their derivatives are very useful multifunctional synthons in organic chemistry [1-5]. Since the pioneering work of Lu and co-workers in 2004, MBH carbonates have triggered much interest among chemistry researchers [6]. The most extensively studied transformation pattern of this type of $\mathrm{MBH}$ derivatives is the allylic substitution with a pronucleophile in the presence of a Lewis basic catalyst [7-9]. Based on the substitution position on $\mathrm{MBH}$ carbonates, the transformations could be divided into the following two styles: substitution at the $\beta$-position through a $\mathrm{S}_{\mathrm{N}} 2$ '- $\mathrm{S}_{\mathrm{N}} 2$ ' cascade or substitution at the $\beta^{\prime}$-position via a single $\mathrm{S}_{\mathrm{N}} 2$ ' route (Scheme 1). Compared with the former route, which is widely employed in asymmetric synthesis to access versatile multifunctional chiral molecules [10-16], the latter route has been often used for the preparation of trisubstituted alkenes [17-20].

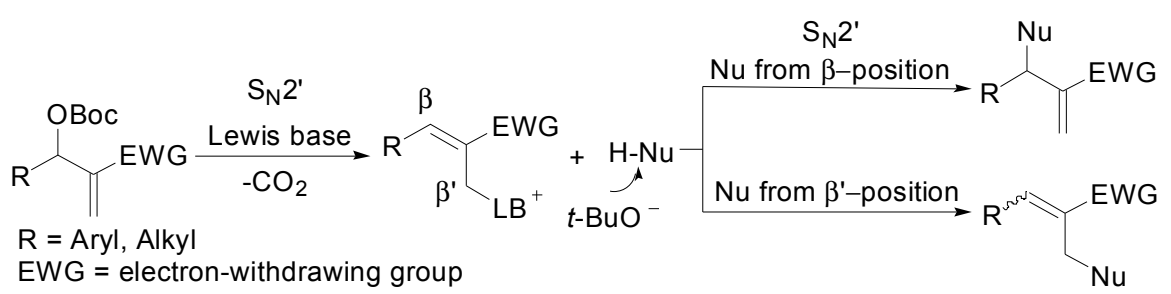

Scheme 1. Allylic substitution reaction of Morita-Baylis-Hillman (MBH) carbonates.

Allylic sulfone derivatives are important intermediates in organic synthesis [21-24]. Recent studies have revealed that these compounds exhibit remarkable biological activities [25]. The use of MBH adducts or their acetates as good starting materials for the trisubstituted allylic sulfones has been reported in some instances [26-34]. Although many sulfonyl precursors including sulfinate [26-29], $p$-toluenesulfonylmethylcyanide [30], arenesulfonyl cyanide [31], sulfinyl chloride [32], sulfonylhydrazide [33] and sulfinic acid [34] have been employed in this type of allylic sulfonylation, sulfinate is undoubtedly a cheap and easily available reagent. However, either a high reaction temperature [26,27] $\left(70-80{ }^{\circ} \mathrm{C}, 6-16 \mathrm{~h}\right)$ or unconventional organic solvent (ionic liquids or polyethylene glycol) [28,29], accompanied with tedious work-up procedures, were required to ensure a high yield of the desired products. Since MBH carbonates usually exhibit much superior reactivity to $\mathrm{MBH}$ acetates, we envisaged that they would be more susceptible to the nucleophilic attack by sulfinate. Herein, we report a new protocol in which MBH carbonates $\mathbf{1}$ and sodium sulfinates 2 undergo a smooth and rapid $S_{N} 2$ ' pathway to realize the trisubstituted allylic sulfones $\mathbf{3}$ under mild and catalyst-free reaction conditions (Scheme 2).

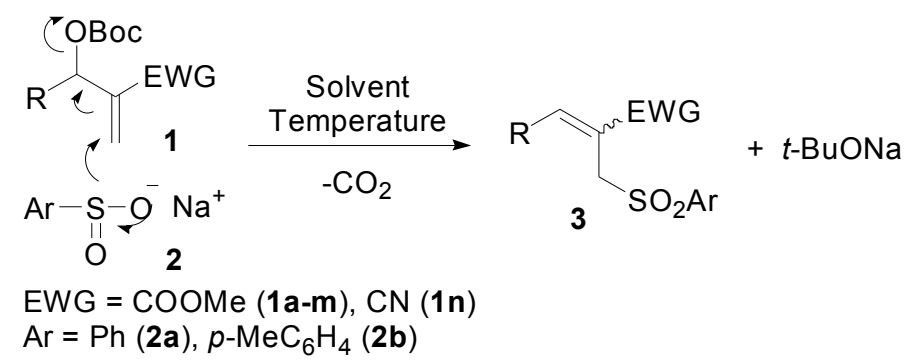

Scheme 2. Allylic sulfonylation of MBH carbonates $\mathbf{1}$ with sodium sulfinates 2. 


\section{Results and Discussion}

\subsection{Optimization Studies}

Preliminary studies were carried out by using $\mathrm{MBH}$ carbonate $1 \mathbf{a}\left(\mathrm{R}=\mathrm{Ph}, \mathrm{EWG}=\mathrm{CO}_{2} \mathrm{Me}\right)$ and sodium benzenesulfinate $(\mathbf{2 a}, \mathrm{Ar}=\mathrm{Ph})$. The screening results are presented in Table 1. Firstly, the model reaction was investigated with different solvents at ambient temperature (Table 1, entries 1-6). Among the solvents tested, toluene and chloroform gave poor conversion (Table 1, entries 1 and 2), and $\mathrm{PhCF}_{3}$ afforded only a trace amount of the final adduct $\mathbf{3 a}$ after $72 \mathrm{~h}$ (Table 1, entry 3). Although 1,2-dichloroethane (DCE) and tetrahydrofuran (THF) afforded 3a in high yield (Table 1, entries 4 and $5)$, acetonitrile was a superior solvent with regard to both conversion rate and product yield ( $92 \%$, Table 1, entry 6). Next, it was found that when the reaction temperature was raised to $40{ }^{\circ} \mathrm{C}$, nearly full conversion could be achieved within a significantly shortened reaction time and the expected product was furnished in $96 \%$ yield (Table 1, entry 7). Finally, the examination of the reaction with a decreased concentration of substrate 1 a revealed no influence on product yield, whereas the reaction time was prolonged (Table 1, entry 8).

Table 1. Optimization of reaction conditions using $\mathrm{MBH}$ carbonate 1a and sodium benzenesulfinate $\mathbf{2} \mathbf{a}^{\text {a }}$.

\begin{tabular}{ccccc}
\hline Entry & Solvent & $\mathbf{T}\left({ }^{\circ} \mathbf{C}\right)$ & Time (h) & Yield (\%) $)^{\mathbf{b , c}}$ \\
\hline 1 & toluene & 25 & 36 & 37 \\
2 & $\mathrm{CHCl}_{3}$ & 25 & 48 & 17 \\
3 & $\mathrm{PhCF}_{3}$ & 25 & 72 & trace \\
4 & $\mathrm{DCE}$ & 25 & 36 & 82 \\
5 & $\mathrm{THF}$ & 25 & 36 & 87 \\
6 & $\mathrm{MeCN}$ & 25 & 36 & 92 \\
7 & $\mathbf{M e C N}$ & $\mathbf{4 0}$ & $\mathbf{2}$ & $\mathbf{9 6}^{\text {d }}$ \\
$8^{\mathrm{e}}$ & $\mathrm{MeCN}$ & 40 & 5 & 96 \\
\hline
\end{tabular}

a Reaction conditions: Unless otherwise noted, reactions were performed with 1a $(0.1 \mathrm{mmol})$ and sodium benzenesulfinate $2 \mathrm{a}(0.15 \mathrm{mmol})$ in solvent $(1 \mathrm{~mL})$ at indicated temperature; ${ }^{\mathrm{b}}$ Isolated yield of two inseparable isomers; ${ }^{c}$ Major isomer of 3a was determined to be $Z$ by comparison of its NMR data with the one reported in literature [35]; ${ }^{\mathrm{d}} Z / E=96: 4$, determined by ${ }^{1} \mathrm{H}-\mathrm{NMR}$ analysis; ${ }^{\mathrm{e}} 2 \mathrm{~mL}$ of solvent was used.

\subsection{Synthesis of Trisubstituted Allylic Sulfones $\mathbf{3 a - 0}$}

On the basis of the above optimized reaction parameters $(0.1 \mathrm{mmol}$ of $\mathrm{MBH}$ carbonate 1a and $0.15 \mathrm{mmol}$ of sodium benzenesulfinate (2a) to perform the reaction in $1 \mathrm{~mL}$ of $\mathrm{MeCN}$ at $40{ }^{\circ} \mathrm{C}$ ), this protocol was then extended to other $\mathrm{MBH}$ carbonates or sulfinates to investigate the scope and limitation of the method. As shown in Table 2, MBH carbonates 1 could generally be converted within $2 \mathrm{~h}$ and corresponding products 3 were obtained in good to excellent yields (71\%-99\%) with good to high selectivity (Z/E from 79:21 to >99:1) (Table 2, entries 1-15). Different substituents on the phenyl group were first explored. The results showed that the electronic nature or position of substituents had minimal influence on reaction efficiency in terms of reaction rate and product yield in general (78\%-98\%, Table 2, entries 1-9). Besides, 1-naphthyl group-substituted $\mathrm{MBH}$ carbonate $\mathbf{1 j}$ was a 
suitable substrate, albeit with lower yield (71\%, Table 2, entry 10). Meanwhile, two heteroaromatic substrates $1 \mathbf{k}$ and $\mathbf{1 1}$ also showed high reactivity, providing 3k and $\mathbf{3 1}$ in high yields $(85 \%$ and $96 \%$, Table 2, entries 11 and 12). It is worth mentioning that the MBH carbonate $\mathbf{1 m}$, which was prepared from an aliphatic aldehyde, could participate in this reaction to produce the desired product $\mathbf{3 m}$ in high yield (91\%, Table 2, entry 13). In addition, MBH carbonate 1n, which was derived from acrylonitrile, could also be transformed in excellent yield under the catalyst-free reaction conditions $(99 \%$, Table 2 , entry 14). To our delight, sodium $p$-toluenesulfinate $\mathbf{2 b}\left(\mathrm{Ar}=p-\mathrm{MeC}_{6} \mathrm{H}_{4}\right)$ was well tolerated and the desired product 30 was provided in $95 \%$ yield (Table 2 , entry 15 ).

Table 2. Substrate scope for allylic sulfonylation of MBH carbonates 1 with sodium sulfinates 2 a.

\begin{tabular}{|c|c|c|c|c|c|}
\hline Entry & $\mathbf{R}$ & EWG & Ar & Yield $(\%)^{b}$ & $Z / E^{\mathrm{c}, \mathrm{d}}$ \\
\hline 1 & $\mathrm{Ph}(\mathbf{1 a})$ & $\mathrm{CO}_{2} \mathrm{Me}$ & $\mathrm{Ph}$ & $96(\mathbf{3 a})$ & $96: 4$ \\
\hline 2 & $o-\mathrm{ClC}_{6} \mathrm{H}_{4}(\mathbf{1 b})$ & $\mathrm{CO}_{2} \mathrm{Me}$ & $\mathrm{Ph}$ & $88(\mathbf{3 b})$ & $95: 5$ \\
\hline 3 & $p-\mathrm{ClC}_{6} \mathrm{H}_{4}(\mathbf{1 c})$ & $\mathrm{CO}_{2} \mathrm{Me}$ & $\mathrm{Ph}$ & $96(3 c)$ & $85: 15$ \\
\hline 4 & $p-\mathrm{NO}_{2} \mathrm{C}_{6} \mathrm{H}_{4}(\mathbf{1 d})$ & $\mathrm{CO}_{2} \mathrm{Me}$ & $\mathrm{Ph}$ & $83(\mathbf{3 d})$ & $79: 21$ \\
\hline 5 & $m-\mathrm{BrC}_{6} \mathrm{H}_{4}(\mathbf{1 e})$ & $\mathrm{CO}_{2} \mathrm{Me}$ & $\mathrm{Ph}$ & $93(\mathbf{3 e})$ & $94: 6$ \\
\hline 6 & $p-\mathrm{MeOC}_{6} \mathrm{H}_{4}(\mathbf{1 f})$ & $\mathrm{CO}_{2} \mathrm{Me}$ & $\mathrm{Ph}$ & 78 (3f) & $88: 12$ \\
\hline 7 & $\mathrm{MeO}^{-1 \mathrm{~g})}$ & $\mathrm{CO}_{2} \mathrm{Me}$ & $\mathrm{Ph}$ & 98 (3g) & $90: 10$ \\
\hline 8 & $m-\mathrm{MeC}_{6} \mathrm{H}_{4}(\mathbf{1 h})$ & $\mathrm{CO}_{2} \mathrm{Me}$ & $\mathrm{Ph}$ & $96(\mathbf{3 h})$ & $88: 12$ \\
\hline 9 & $p-\mathrm{MeC}_{6} \mathrm{H}_{4}(\mathbf{1 i})$ & $\mathrm{CO}_{2} \mathrm{Me}$ & $\mathrm{Ph}$ & $92(\mathbf{3 i})$ & $96: 4$ \\
\hline 10 & 1-naphthyl (1j) & $\mathrm{CO}_{2} \mathrm{Me}$ & $\mathrm{Ph}$ & $71(\mathbf{3} \mathbf{j})$ & $96: 4$ \\
\hline 11 & 2-furyl (1k) & $\mathrm{CO}_{2} \mathrm{Me}$ & $\mathrm{Ph}$ & $85(3 k)$ & $>99: 1$ \\
\hline 12 & 2-thienyl (11) & $\mathrm{CO}_{2} \mathrm{Me}$ & $\mathrm{Ph}$ & $96(31)$ & $81: 19$ \\
\hline 13 & $n$-propyl (1 $\mathbf{m})$ & $\mathrm{CO}_{2} \mathrm{Me}$ & $\mathrm{Ph}$ & $91(\mathbf{3 m})$ & $82: 18$ \\
\hline 14 & $\mathrm{Ph}(\mathbf{1 n})$ & $\mathrm{CN}$ & $\mathrm{Ph}$ & $99(3 n)$ & $<1: 99$ \\
\hline 15 & $\mathrm{Ph}(\mathbf{1 a})$ & $\mathrm{CO}_{2} \mathrm{Me}$ & $p-\mathrm{MeC}_{6} \mathrm{H}_{4}$ & $95(\mathbf{3 o})$ & $84: 16$ \\
\hline
\end{tabular}

${ }^{a}$ Reaction conditions: Unless otherwise noted, reactions were performed with MBH carbonate $1(0.1 \mathrm{mmol})$ and sodium sulfinate $2(0.15 \mathrm{mmol})$ in $\mathrm{MeCN}(1 \mathrm{~mL})$ at $40{ }^{\circ} \mathrm{C}$ for $2 \mathrm{~h} ;{ }^{b}$ Isolated yield of two inseparable isomers; ${ }^{\mathrm{c}}$ Olefin geometry was assigned by analogy with that of $\mathbf{3 a} ;{ }^{\mathrm{d}} \mathrm{Z} / \mathrm{E}$ ratio was determined by ${ }^{1} \mathrm{H}-\mathrm{NMR}$ analysis.

\section{Experimental Section}

\subsection{General Information}

TLC was performed on glass-backed silica plates. Flash column chromatography was performed using silica gel (200-300 mesh) eluting with ethyl acetate and petroleum ether. UV light was used to visualize products. ${ }^{1} \mathrm{H}-\mathrm{NMR}$ spectra were recorded at $400 \mathrm{MHz}$, and ${ }^{13} \mathrm{C}-\mathrm{NMR}$ spectra were recorded at $100 \mathrm{MHz}$ (Avance 400, Bruker, Faellanden, Switzerland). Tetramethylsilane was used as the internal standard. Chemical shifts are reported in ppm downfield from the solvent signal $\left(\mathrm{CDCl}_{3}, \delta=7.27 \mathrm{ppm}\right)$ for ${ }^{1} \mathrm{H}-\mathrm{NMR}$ and relative to the central $\mathrm{CDCl}_{3}$ resonance $(\delta=77.0 \mathrm{ppm})$ for ${ }^{13} \mathrm{C}-\mathrm{NMR}$ spectroscopy. Coupling constants are given in Hz. ESI-HRMS was recorded on a Waters SYNAPT G2 (Milford, MA, USA). In experiments requiring dry solvents, DCE, chloroform and toluene were distilled from $\mathrm{CaH}_{2}$. $\mathrm{PhCF}_{3}$ was stored over $4 \AA$ molecular sieves. THF was dried over sodium metal. Acetonitrile was 
dried over $\mathrm{P}_{2} \mathrm{O}_{5}$. All other chemicals were used without purification as commercially available. $\mathrm{MBH}$ carbonates were prepared by the reported procedure [36].

\subsection{General Procedure for Preparation of Trisubstituted Allylic Sulfones 3a-0}

MBH carbonate $1(0.1 \mathrm{mmol})$ and sodium sulfinate $2(0.15 \mathrm{mmol})$ were mixed in MeCN $(1 \mathrm{~mL})$ and heated at $40{ }^{\circ} \mathrm{C}$ for $2 \mathrm{~h}$. Then, the reaction mixture was concentrated under reduced pressure and the residue was diluted with toluene and purified by flash column chromatography on silica gel (petroleum ether/EtOAc) to afford the desired product 3a-o. Products 3a [33], 3d [26], 3f [26], 3k [26], 31 [27], 3n [33] and 3o [26] are known compounds.

(Z)-Methyl 3-phenyl-2-[(phenylsulfonyl)methyl] acrylate (3a). Colourless liquid; 96\% yield; Z/E = 96:4; ${ }^{1} \mathrm{H}-\mathrm{NMR}: \delta=7.95(\mathrm{~s}, 1 \mathrm{H}), 7.86(\mathrm{~d}, J=8.0 \mathrm{~Hz}, 2 \mathrm{H}), 7.63-7.59(\mathrm{~m}, 1 \mathrm{H}), 7.52-7.47(\mathrm{~m}, 4 \mathrm{H}), 7.39-7.37$ $(\mathrm{m}, 3 \mathrm{H}), 4.49$ (s, 2H), 3.60 (s, 3H) ppm; ${ }^{13} \mathrm{C}-\mathrm{NMR}: \delta=167.0,146.6,139.6,133.9,133.8,129.9,129.4$, 129.2, 129.0, 128.7, 121.1, 55.3, 52.5 ppm; ESI-HRMS: calcd. For $\mathrm{C}_{17} \mathrm{H}_{16} \mathrm{O}_{4} \mathrm{~S}+\mathrm{Na} 339.0667$, found 339.0661 .

(Z)-Methyl 3-(2-chlorophenyl)-2-[(phenylsulfonyl)methyl]acrylate (3b). Colourless liquid; 88\% yield; $Z / E=95: 5 ;{ }^{1} \mathrm{H}-\mathrm{NMR}: \delta=7.94(\mathrm{~m}, 1 \mathrm{H}), 7.75(\mathrm{~d}, J=8.0 \mathrm{~Hz}, 2 \mathrm{H}), 7.55-7.53(\mathrm{~m}, 2 \mathrm{H}), 7.43-7.40(\mathrm{~m}$, 2H), 7.30-7.27 (m, 1H), 7.23-7.21 (m, 2H), $4.31(\mathrm{~s}, 2 \mathrm{H}), 3.55(\mathrm{~s}, 3 \mathrm{H}) \mathrm{ppm} ;{ }^{13} \mathrm{C}-\mathrm{NMR}: \delta=165.5$, 142.4, 138.5, 133.3, 133.0, 131.5, 129.9, 129.2, 129.0, 128.3, 128.1, 127.7, 126.3, 122.3, 54.2, 51.8 ppm; ESI-HRMS: calcd. For $\mathrm{C}_{17} \mathrm{H}_{15} \mathrm{ClO}_{4} \mathrm{~S}+\mathrm{Na} 373.0277$, found 373.0273 .

(Z)-Methyl 3-(4-chlorophenyl)-2-[(phenylsulfonyl)methyl]acrylate (3c). Colourless liquid; 96\% yield; $Z / E=85: 15 ;{ }^{1} \mathrm{H}-\mathrm{NMR}: \delta=7.89(\mathrm{~s}, 1 \mathrm{H}), 7.85(\mathrm{~d}, J=8.0 \mathrm{~Hz}, 2 \mathrm{H}), 7.64-7.61(\mathrm{~m}, 1 \mathrm{H}), 7.53-7.49(\mathrm{~m}, 2 \mathrm{H})$, $7.46(\mathrm{~d}, J=8.0 \mathrm{~Hz}, 2 \mathrm{H}), 7.35(\mathrm{~d}, J=8.0 \mathrm{~Hz}, 2 \mathrm{H}), 4.44(\mathrm{~s}, 2 \mathrm{H}), 3.57$ (s, 3H) ppm; ${ }^{13} \mathrm{C}-\mathrm{NMR}: \delta=165.6$, 144.0, 138.3, 134.9, 132.9, 131.1, 129.6, 128.1, 127.5, 120.4, 54.1, 51.5 ppm; ESI-HRMS: calcd. For $\mathrm{C}_{17} \mathrm{H}_{15} \mathrm{ClO}_{4} \mathrm{~S}+\mathrm{H} 351.0458$, found 351.0459 .

(Z)-Methyl 3-(4-nitrophenyl)-2-[(phenylsulfonyl)methyl]acrylate (3d). Pale yellow solid; 83\% yield; $Z / E=79: 21 ;{ }^{1} \mathrm{H}-\mathrm{NMR}: \delta=8.24(\mathrm{~d}, J=8.0 \mathrm{~Hz}, 2 \mathrm{H}), 7.98(\mathrm{~s}, 1 \mathrm{H}), 7.86(\mathrm{~d}, J=8.0 \mathrm{~Hz}, 2 \mathrm{H}), 7.69-7.64(\mathrm{~m}$, $3 \mathrm{H}), 7.55-7.52(\mathrm{~m}, 2 \mathrm{H}), 4.40(\mathrm{~s}, 2 \mathrm{H}), 3.63(\mathrm{~s}, 3 \mathrm{H}) \mathrm{ppm} ;{ }^{13} \mathrm{C}-\mathrm{NMR}: \delta=165.0,142.5,139.0,133.1$, $128.8,128.2,127.7,127.5,123.2,122.9,122.4,53.9,51.7$ ppm; ESI-HRMS: calcd. For $\mathrm{C}_{17} \mathrm{H}_{15} \mathrm{NO}_{6} \mathrm{~S}+\mathrm{Na} 384.0518$, found 384.0518 .

(Z)-Methyl 3-(3-bromophenyl)-2-[(phenylsulfonyl)methyl]acrylate (3e). Pale yellow solid; 93\% yield; $Z / E=94: 6$; ${ }^{1} \mathrm{H}-\mathrm{NMR}: \delta=7.85-7.83(\mathrm{~m}, 3 \mathrm{H}), 7.68-7.64(\mathrm{~m}, 1 \mathrm{H}), 7.53-7.42(\mathrm{~m}, 5 \mathrm{H}), 7.28-7.24(\mathrm{~m}$, $1 \mathrm{H}), 4.46(\mathrm{~s}, 2 \mathrm{H}), 3.67(\mathrm{~s}, 3 \mathrm{H}) \mathrm{ppm} ;{ }^{13} \mathrm{C}-\mathrm{NMR}: \delta=166.6,144.6,139.1,135.7,134.0,132.6,131.9$, $130.4,129.3,128.7,127.4,123.0,122.7,55.0,52.7$ ppm; ESI-HRMS: calcd. For $\mathrm{C}_{17} \mathrm{H}_{15} \mathrm{BrO}_{4} \mathrm{~S}+\mathrm{Na}$ 416.9772, found 416.9772 .

(Z)-Methyl 3-(4-methoxyphenyl)-2-[(phenylsulfonyl)methyl] acrylate (3f). Colourless liquid; 78\% yield; $Z / E=88: 12 ;{ }^{1} \mathrm{H}-\mathrm{NMR}: \delta=7.92(\mathrm{~s}, 1 \mathrm{H}), 7.89(\mathrm{~d}, J=8.0 \mathrm{~Hz}, 2 \mathrm{H}), 7.62-7.58(\mathrm{~m}, 3 \mathrm{H}), 7.54-7.50(\mathrm{~m}$, 2H), $6.93(\mathrm{~d}, J=8.0 \mathrm{~Hz}, 2 \mathrm{H}), 4.52(\mathrm{~s}, 2 \mathrm{H}), 3.85(\mathrm{~s}, 3 \mathrm{H}), 3.51(\mathrm{~s}, 3 \mathrm{H}) \mathrm{ppm} ;{ }^{13} \mathrm{C}-\mathrm{NMR}: \delta=167.3,161.3$, 
146.5, 139.6, 133.8, 131.7, 129.1, 128.7, 126.3, 118.1, 114.4, 55.6, 55.5, 52.3 ppm; ESI-HRMS: calcd. For $\mathrm{C}_{18} \mathrm{H}_{18} \mathrm{O}_{5} \mathrm{~S}+\mathrm{Na} 369.0773$, found 369.0771 .

(Z)-Methyl 3-(3,4-dimethoxyphenyl)-2-[(phenylsulfonyl)methyl]acrylate (3g). Viscous liquid; 98\% yield; $Z / E=90: 10 ;{ }^{1} \mathrm{H}-\mathrm{NMR}: \delta=7.93(\mathrm{~s}, 1 \mathrm{H}), 7.90(\mathrm{~d}, J=8.0 \mathrm{~Hz}, 2 \mathrm{H}), 7.65-7.62(\mathrm{~m}, 1 \mathrm{H}), 7.55-7.51$ (m, 2H), $7.43(\mathrm{~s}, 1 \mathrm{H}), 7.20-7.17(\mathrm{~m}, 1 \mathrm{H}), 6.90(\mathrm{~d}, J=8.0 \mathrm{~Hz}, 1 \mathrm{H}), 4.53(\mathrm{~s}, 2 \mathrm{H}), 3.97(\mathrm{~s}, 3 \mathrm{H}), 3.93$ (s, 3H), 3.50 (s, 3H), ppm; ${ }^{13} \mathrm{C}-\mathrm{NMR}: \delta=166.1,149.8,148.1,145.8,138.6,132.7,128.0,127.6,125.5$, 122.7, 117.2, 111.5, 110.1, 55.3, 55.0, 54.8, 51.2 ppm; ESI-HRMS: calcd. For $\mathrm{C}_{19} \mathrm{H}_{20} \mathrm{O} 6 \mathrm{~S}+\mathrm{K} 415.0618$, found 415.0618 .

(Z)-Methyl 2-[(phenylsulfonyl)methyl]-3-(m-tolyl)acrylate (3h). Colourless liquid; 96\% yield; $Z / E=88: 12$; ${ }^{1} \mathrm{H}-\mathrm{NMR}: \delta=7.88(\mathrm{~s}, 1 \mathrm{H}), 7.81(\mathrm{~d}, J=8.0 \mathrm{~Hz}, 2 \mathrm{H}), 7.59-7.54(\mathrm{~m}, 1 \mathrm{H}), 7.47-7.43(\mathrm{~m}$, 2H), 7.24-7.20 (m, 2H), 7.16-7.13 (m, 2H), $4.47(\mathrm{~s}, 2 \mathrm{H}), 3.58(\mathrm{~s}, 3 \mathrm{H}), 2.31(\mathrm{~s}, 3 \mathrm{H}) \mathrm{ppm}$; ${ }^{13} \mathrm{C}-\mathrm{NMR}$ : $\delta=165.9,145.5,138.3,137.4,132.6,129.5,128.8,127.7,127.5,125.1,119.7,54.1,51.4,20.3$ ppm; ESI-HRMS: calcd. For $\mathrm{C}_{18} \mathrm{H}_{18} \mathrm{O}_{4} \mathrm{~S}+\mathrm{Na} 353.0823$, found 353.0827 .

(Z)-Methyl 2-[(phenylsulfonyl)methyl]-3-(p-tolyl)acrylate (3i). Colourless liquid; 92\% yield; $Z / E=96: 4 ;{ }^{1} \mathrm{H}-\mathrm{NMR}: \delta=7.93(\mathrm{~s}, 1 \mathrm{H}), 7.87(\mathrm{~d}, J=8.0 \mathrm{~Hz}, 2 \mathrm{H}), 7.63-7.60(\mathrm{~m}, 1 \mathrm{H}), 7.52-7.48(\mathrm{~m}, 2 \mathrm{H})$, $7.43(\mathrm{~d}, J=8.0 \mathrm{~Hz}, 2 \mathrm{H}), 7.19(\mathrm{~d}, J=8.0 \mathrm{~Hz}, 2 \mathrm{H}), 4.50(\mathrm{~s}, 2 \mathrm{H}), 3.56(\mathrm{~s}, 3 \mathrm{H}), 2.38$ (s, 3H) ppm; ${ }^{13} \mathrm{C}-\mathrm{NMR}: \delta=166.0,145.6,139.3,138.5,132.7,129.8,128.5,128.5,128.0,127.6,118.7,54.3,51.3$, 20.4 ppm; ESI-HRMS: calcd. For $\mathrm{C}_{18} \mathrm{H}_{18} \mathrm{O} 4 \mathrm{~S}+\mathrm{Na} 353.0823$, found 353.0820.

(Z)-Methyl 3-(naphthalen-2-yl)-2-[(phenylsulfonyl)methyl] acrylate (3j). Colourless liquid; 71\% yield; $Z / E=96: 4 ;{ }^{1} \mathrm{H}-\mathrm{NMR}: \delta=8.35(\mathrm{~s}, 1 \mathrm{H}), 7.75(\mathrm{~d}, J=8.0 \mathrm{~Hz}, 2 \mathrm{H}), 7.62-7.59(\mathrm{~m}, 3 \mathrm{H}), 7.46-7.33(\mathrm{~m}, 4 \mathrm{H})$, 7.29-7.26 (m, 1H), 7.21-7.17 (m, 2H), $4.38(\mathrm{~s}, 2 \mathrm{H}), 3.66(\mathrm{~s}, 3 \mathrm{H}) \mathrm{ppm} ;{ }^{13} \mathrm{C}-\mathrm{NMR}: \delta=166.7,144.6$, 139.2, 133.4, 133.3, 131.0, 130.7, 129.8, 128.9, 128.6, 128.1, 126.7, 126.4, 126.4, 125.3, 124.3, 123.5, 55.1, 52.6 ppm; ESI-HRMS: calcd. For $\mathrm{C}_{21} \mathrm{H}_{18} \mathrm{O} 4 \mathrm{~S}+\mathrm{Na} 389.0823$, found 389.0825.

(Z)-Methyl 3-(furan-2-yl)-2-[(phenylsulfonyl)methyl] acrylate (3k). Semi-solid; 85\% yield; Z/E > 99:1; ${ }^{1} \mathrm{H}-\mathrm{NMR}: \delta=7.84(\mathrm{~s}, 1 \mathrm{H}), 7.83-7.82(\mathrm{~m}, 1 \mathrm{H}), 7.53-7.50(\mathrm{~m}, 2 \mathrm{H}), 7.44-7.39(\mathrm{~m}, 3 \mathrm{H}), 6.67(\mathrm{~d}$, $J=4.0 \mathrm{~Hz}, 1 \mathrm{H}), 6.40(\mathrm{~m}, 1 \mathrm{H}), 4.79(\mathrm{~s}, 2 \mathrm{H}), 3.59$ (s, 3H) ppm; ${ }^{13} \mathrm{C}-\mathrm{NMR}: \delta=166.9,150.0,145.8,139.4$, 133.6, 130.7, 128.7, 128.7, 119.1, 115.5, 112.3, 55.5, 52.4 ppm; ESI-HRMS: calcd. For $\mathrm{C}_{15} \mathrm{H}_{14} \mathrm{O}_{5} \mathrm{~S}+\mathrm{H}$ 307.0640, found 307.0649.

(Z)-Methyl 2-[(phenylsulfonyl)methyl]-3-(thiophen-2-yl)acrylate (31). Light brown liquid; 96\% yield; $Z / E=81: 19 ;{ }^{1} \mathrm{H}-\mathrm{NMR}: \delta=8.03(\mathrm{~s}, 1 \mathrm{H}), 7.88(\mathrm{~d}, J=8.0 \mathrm{~Hz}, 1 \mathrm{H}), 7.60-7.56(\mathrm{~m}, 1 \mathrm{H}), 7.53-7.46(\mathrm{~m}$, $5 \mathrm{H}), 7.08-7.06(\mathrm{~m}, 1 \mathrm{H}), 4.61(\mathrm{~s}, 2 \mathrm{H}), 3.50(\mathrm{~s}, 3 \mathrm{H}) \mathrm{ppm} ;{ }^{13} \mathrm{C}-\mathrm{NMR}: \delta=165.7,138.4,137.3,135.7$, 133.2, 132.8, 129.9, 128.0, 127.6, 126.8, 115.1, 55.0, 51.3 ppm; ESI-HRMS: calcd. For $\mathrm{C}_{15} \mathrm{H}_{14} \mathrm{O}_{4} \mathrm{~S}_{2}+\mathrm{H}$ 323.0412, found 323.0417 .

(Z)-Methyl 2-[(phenylsulfonyl)methyl]hex-2-enoate (3m). viscous liquid; 91\% yield; $Z / E=82: 18$; ${ }^{1} \mathrm{H}-\mathrm{NMR}: \delta=7.86-7.81(\mathrm{~m}, 2 \mathrm{H}), 7.64-7.61(\mathrm{~m}, 1 \mathrm{H}), 7.54-7.51(\mathrm{~m}, 2 \mathrm{H}), 7.12(\mathrm{t}, J=8.0 \mathrm{~Hz}, 1 \mathrm{H}), 4.24$ (s, 2H), $3.48(\mathrm{~s}, 3 \mathrm{H}), 2.20-2.14(\mathrm{~m}, 2 \mathrm{H}), 1.49-1.40(\mathrm{~m}, 2 \mathrm{H}), 0.91(\mathrm{t}, J=8.0 \mathrm{~Hz}, 3 \mathrm{H}) \mathrm{ppm} ;{ }^{13} \mathrm{C}-\mathrm{NMR}$ : 
$\delta=166.2,151.9,139.0,133.9,129.2,128.9,120.8,54.2,52.1,31.6,21.7,14.0$ ppm; ESI-HRMS: calcd. For $\mathrm{C}_{14} \mathrm{H}_{18} \mathrm{O}_{4} \mathrm{~S}+\mathrm{Na} 305.0823$, found 305.0829 .

(E)-3-Phenyl-2-[(phenylsulfonyl)methyl]acrylonitrile (3n). Colourless liquid; 99\% yield; Z/E < 1:99; ${ }^{1} \mathrm{H}-\mathrm{NMR}: \delta=7.94-7.92(\mathrm{~m}, 2 \mathrm{H}), 7.74-7.68(\mathrm{~m}, 3 \mathrm{H}), 7.63-7.59(\mathrm{~m}, 2 \mathrm{H}), 7.47-7.41(\mathrm{~m}, 3 \mathrm{H}), 7.09$ (s, 1H), 4.05 (s, 2H) ppm; ${ }^{13} \mathrm{C}-\mathrm{NMR}: \delta=151.9,137.6,134.7,132.5,131.7,129.6,129.3,129.1,128.8$, 117.1, 98.0, 61.4 ppm; ESI-HRMS: calcd. For $\mathrm{C}_{16} \mathrm{H}_{13} \mathrm{NO}_{2} \mathrm{~S}+\mathrm{Na}$ 306.0565, found 306.0566.

(Z)-Methyl 3-phenyl-2-(tosylmethyl)acrylate (3o). Colourless liquid; 95\% yield; $Z / E=84: 16$; ${ }^{1} \mathrm{H}-\mathrm{NMR}: \delta=7.93(\mathrm{~s}, 1 \mathrm{H}), 7.71(\mathrm{~d}, J=8.0 \mathrm{~Hz}, 2 \mathrm{H}), 7.47(\mathrm{~m}, 2 \mathrm{H}), 7.37(\mathrm{~m}, 3 \mathrm{H}), 7.27(\mathrm{~d}, J=8.0 \mathrm{~Hz}$, $2 \mathrm{H}), 4.48(\mathrm{~s}, 2 \mathrm{H}), 3.62(\mathrm{~s}, 3 \mathrm{H}), 2.42(\mathrm{~s}, 3 \mathrm{H}) \mathrm{ppm} ;{ }^{13} \mathrm{C}-\mathrm{NMR}: \delta=167.1,146.3,144.8,136.3,133.8$, 129.7, 129.3, 128.8, 128.6, 121.2, 55.2, 52.5, 21.7 ppm; ESI-HRMS: calcd. For $\mathrm{C}_{18} \mathrm{H}_{18} \mathrm{O}_{4} \mathrm{~S}+\mathrm{Na}$ 353.0823 , found 353.0827 .

\section{Conclusions}

In summary, we have established a method for the allylic sulfonylation of MBH carbonates with sodium sulfinates under catalyst-free reaction conditions. A series of functionalized trisubstituted allylic sulfones were rapidly generated in good to excellent yields (71\%-99\%) with good to high selectivity (Z/E from 79:21 to $>99: 1)$. Compared with known synthetic methods, the current protocol features mild reaction temperature $\left(40^{\circ} \mathrm{C}\right.$ ), high efficiency (full conversion within $2 \mathrm{~h}$ ) and easily available reagents. Thus, it should provide an efficient and facile access to the trisubstituted allylic sulfones. Further studies on expanding the substrate scope and chemical transformations of the trisubstituted allylic sulfones are currently underway.

\section{Supplementary Materials}

Supplementary materials can be accessed at: http://www.mdpi.com/1420-3049/20/05/8213/s1.

\section{Acknowledgments}

We are grateful for financial support from the National Natural Science Foundation of China (21302163), Scientific Research Foundation of Yunnan Education Office (2013Z037), Youth Scientific Research Foundation of Yunnan Minzu University (2012QN03) and Graduate Students Innovation Foundation of Yunnan Minzu University (2014YJY85).

\section{Author Contributions}

L.J. and M.-L.Y. designed research; L.J. wrote the paper as well; Y.-G.L. and J.-F.Z. performed the experiments; Y.-M.C. analyzed the data; H.-L.L. revised the manuscript. All authors read and approved the final manuscript.

\section{Conflicts of Interest}

The authors declare no conflict of interest. 


\section{References and Notes}

1. Basavaiah, D.; Reddy, B.S.; Badsara, S.S. Recent contributions from the Baylis-Hillman reaction to organic chemistry. Chem. Rev. 2010, 110, 5447-5674.

2. Ma, G.-N.; Jiang, J.-J.; Shi, M.; Wei, Y. Recent extensions of the Morita-Baylis-Hillman reaction. Chem. Commun. 2009, 5496-5514.

3. Declerck, V.; Martinez, J.; Lamaty, F. Aza-Baylis-Hillman reaction. Chem. Rev. 2009, 109, 1-48.

4. Gowrisankar, S.; Lee, H.S.; Kim, S.H.; Lee, K.Y.; Kim, J.N. Recent advances in the Pd-catalyzed chemical transformations of Baylis-Hillman adducts. Tetrahedron 2009, 65, 8769-8780.

5. Wei, Y.; Shi, M. Multifunctional chiral phosphine organocatalysts in catalytic asymmetric Morita-Baylis-Hillman and related reactions. Acc. Chem. Res. 2010, 43, 1005-1018.

6. Du, Y.; Han, X.; Lu, X. Alkaloids-catalyzed regio- and enantioselective allylic nucleophilic substitution of tert-butyl carbonate of the Morita-Baylis-Hillman products. Tetrahedron Lett. 2004, 45, 4967-4971.

7. Liu, T.-Y.; Xie, M.; Chen, Y.-C. Organocatalytic asymmetric transformations of modified Morita-Baylis-Hillman adducts. Chem. Soc. Rev. 2012, 41, 4101-4112.

8. Rios, R. Organocatalytic enantioselective methodologies using Morita-Baylis-Hillman carbonates and acetates. Catal. Sci. Technol. 2012, 2, 267-278.

9. Wei, Y.; Shi, M. Recent advances in organocatalytic asymmetric Morita-Baylis-Hillman/azaMorita-Baylis-Hillman reactions. Chem. Rev. 2013, 113, 6659-6690.

10. Zhan, G.; Zhou, Q.-Q.; Du, W.; Chen, Y.-C. Enantioselective $N$-allylic alkylation of $\mathrm{N}$-propargylsulfonamides with Morita-Baylis-Hillman carbonates and sequential electrophilic cyclization. Synthesis 2014, 46, 3383-3393.

11. Yang, H.-B.; Zhao, Y.-Z.; Sang, R.; Shi, M. (DHQ) 2 AQN-Catalyzed asymmetric substitution of isatin-derived hydrazones with $O$-BoC-protected Morita-Baylis-Hillman adducts: A strategy for synthesizing enantioenriched Azo compounds incorporating an oxindole scaffold. J. Org. Chem. 2014, 79, 3519-3528.

12. Companyó, X.; Geant, P.-Y.; Mazzanti, A.; Moyano, A.; Rios, R. Catalytic asymmetric one-pot synthesis of $\alpha$-methylene- $\gamma$-lactams. Tetrahedron 2014, 70, 75-82.

13. Yan, Y.; En, D.; Zhuang, Z.; Guo, Y.; Liao, W.-W. Synthesis of densely functionalized $\alpha$-methylene $\gamma$-butyrolactones via an organ Catalytic one-pot allylic-alkylation-cyclization reaction. Tetrahedron Lett. 2014, 55, 479-482.

14. Mao, H.; Lin, A.; Shi, Y.; Mao, Z.; Zhu, X.; Li, W.; Hu, H.; Cheng, Y.; Zhu, C. Construction of enantiomerically enriched diazo compounds using diazo esters as nucleophiles: Chiral Lewis base catalysis. Angew. Chem. Int. Ed. 2013, 52, 6288-6292.

15. Wang, Q.-G.; Zhou, Q.-Q.; Deng, J.-G.; Chen, Y.-C. An asymmetric allylic alkylation-smiles rearrangement sulfinate addition sequence to construct chiral cyclic sulfones. Org. Lett. 2013, 15, 4786-4789.

16. Companyó, X.; Mazzanti, A.; Moyano, A.; Janecka, A.; Rios, R. First one-pot organocatalytic synthesis of $\alpha$-methylene- $\gamma$-lactones. Chem. Commun. 2013, 49, 1184-1186.

17. Drewes, S.E.; Roos, G.H.P. Synthetic potential of the tertiary-amine-catalysed reaction of activated vinyl carbanions with aldehydes. Tetrahedron 1988, 44, 4653-4670. 
18. Basavaiah, D.; Rao, P.D.; Hyma, R.S. The Baylis-Hillman reaction: A novel carbon-carbon bond forming reaction. Tetrahedron 1996, 52, 8001-8062.

19. Li, G.; Wei, H.-X.; Gao, J.J.; Caputo, T.D. TiCl4-Mediated Baylis-Hillman and aldol reactions without the direct use of a Lewis base. Tetrahedron Lett. 2000, 41, 1-5.

20. Basavaiah, D.; Pandiaraju, S.; Padmaja, K. The Friedel-Crafts chemistry: Acetates of the Baylis-Hillman adducts as novel stereodefined $\beta$-electrophiles. Synlett 1996, 393-395.

21. Quiclet-Sire, B.; Seguin, S.; Zard, S.Z. A new radical allylation reaction of dithiocarbonates. Angew. Chem. Int. Ed. 1998, 37, 2864-2866.

22. Kim, S.; Lim, C.J. Tin-free radical-mediated C-C-bond formations with alkyl allyl sulfones as radical precursors. Angew. Chem. Int. Ed. 2002, 41, 3265-3267.

23. Wróbel, Z. Silane-mediated direct condensation of nitroarenes with cinnamyl-type sulfones. The way to 2-aryl-4-X-quinolines and their hetero analogs. Tetrahedron 1998, 54, 2607-2618.

24. Jiang, L.; Lei, Q.; Huang, X.; Cui, H.-L.; Zhou, X.; Chen, Y.-C. Lewis base assisted Brønsted base catalysis: Direct regioselective asymmetric vinylogous alkylation of allylic sulfones. Chem. Eur. J. 2011, 17, 9489-9493.

25. Neamati, N.; Kabalka, G.W.; Venkataiah, B.; Dayam, R. Small Molecules for Treating Cancer and Abnormal Cell Proliferation Disorders. U.S. Patent WO 2,007,081,966 A3, 9 January 2007.

26. Wang, Q.; Sheng, S.-R.; Lin, S.-Y.; Guo, L.; Wei, M.-H.; Huang, X. Liquid-phase synthesis of methyl (2Z)-2-arylsulfonylmethyl-2-alkenoates from PEG-supported $\alpha$-phenylselenopropionate. Chin. J. Chem. 2007, 25, 1027-1030.

27. Karnakar, K.; Shankar, J.; Murthy, S.N.; Nageswar, Y.V.D. Synthesis of allyl aryl sulfone derivatives from Baylis-Hillman acetates in water. Helv. Chim. Acta 2011, 94, 875-880.

28. Kabalka, G.W.; Venkataiah, B.; Dong, G. Preparation of substituted allyl acetates and sulfones from Baylis-Hillman adducts in ionic liquid media. Tetrahedron Lett. 2003, 44, 4673-4675.

29. Chandrasekhar, S.; Saritha, B.; Jagadeshwer, V.; Narsihmulu, C.; Vijay, D.; Sarma, G.D.; Jagadeesh, B. Hydroxy-assisted catalyst-free Michael addition-dehydroxylation of Baylis-Hillman adducts in poly(ethylene glycol). Tetrahedron Lett. 2006, 47, 2981-2984.

30. Srivastava, V.P.; Yadav, L.D.S. Direct sulfonylation of Baylis-Hillman alcohols and diarylmethanols with TosMIC in ionic liquid-[Hmim] $] \mathrm{HSO}_{4}$ : An unexpected reaction. Tetrahedron Lett. 2011, 52, 4622-4626.

31. Reddy, L.R.; Hu, B.; Prashad, M.; Prasad, K. An unexpected reaction of arenesulfonyl cyanides with allylic alcohols: Preparation of trisubstituted allyl sulfones. Angew. Chem. Int. Ed. 2009, 48, 172-174.

32. Li, H.-H.; Dong, D.-J.; Jin, Y.-H.; Tian, S.-K. An expeditious entry to benzylic and allylic sulfones through byproduct-catalyzed reaction of alcohols with sulfinyl chlorides. J. Org. Chem. 2009, 74, 9501-9504.

33. Li, X.; Xu, X.; Tang, Y. Tetrabutylammonium iodide catalyzed allylic sulfonylation of Baylis-Hillman acetates with sulfonylhydrazides in water. Org. Biomol. Chem. 2013, 11, 1739-1742.

34. Liu, C.-R.; Li, M.-B.; Cheng, D.-J.; Yang, C.-F.; Tian, S.-K. Catalyst-free alkylation of sulfinic acids with sulfonamides via $\mathrm{sp}^{3} \mathrm{C}-\mathrm{N}$ bond cleavage at room temperature. Org. Lett. 2009, 11, 2543-2545. 
35. The NMR characteristic data of 3a are consistent with that of ( $Z$ )-methyl 3-phenyl-2((phenylsulfonyl)methyl)acrylate, which were reported in the ESI section of Ref. [33].

36. Feng, J.; Lu, X.; Kong, A.; Han, X. A highly regio- and stereo-selective [3+2] annulation of allylic compounds and 2-substituted 1,1-dicyanoalkenes through a catalytic carbon-phosphorus ylide reaction. Tetrahedron 2007, 63, 6035-6041.

Sample Availability: Samples of the compounds 3a-o are available from the authors.

(C) 2015 by the authors; licensee MDPI, Basel, Switzerland. This article is an open access article distributed under the terms and conditions of the Creative Commons Attribution license (http://creativecommons.org/licenses/by/4.0/). 